PROCEEDINGS OF THE

AMERICAN MATHEMATICAL SOCIETY

Volume 134, Number 9, September 2006, Pages 2593-2598

S 0002-9939(06)08284-0

Article electronically published on February 17, 2006

\title{
AN INVARIANT FOR UNBOUNDED OPERATORS
}

\author{
VLADIMIR MANUILOV AND SERGEI SILVESTROV
}

(Communicated by David R. Larson)

\begin{abstract}
For a class of unbounded operators, a deformation of a Bott projection is used to construct an integer-valued invariant measuring deviation of the non-commutative deformations from the commutative originals, and its interpretation in terms of $K$-theory of $C^{*}$-algebras is given. Calculation of this invariant for specific important classes of unbounded operators is also presented.
\end{abstract}

We consider a class of unbounded operators on a Hilbert space that can be viewed as deformations of the complex coordinate on a complex plane. In order to distinguish these deformations, we introduce an integer-valued invariant for such operators using the deformed Bott projection and calculate it for some examples. We also give a $K$-theoretical interpretation of this invariant.

Let $x$ be an unbounded (i.e. linear densely defined) operator on a Hilbert space $H$ with the following properties:

(i) operators $x^{*} x$ and $x x^{*}$ are well defined unbounded self-adjoint operators on $H$;

(ii) the accumulation points of $\operatorname{Sp} x^{*} x$ are 0 and $\infty$, and outside these two points $\operatorname{Sp} x^{*} x$ is discrete and of finite multiplicity;

(iii) operators $x^{*} x$ and $x x^{*}$ commute as unbounded operators (i.e. their spectral measures do).

Let $x=u h$ be a polar decomposition with a partial isometry $u$ and an unbounded self-adjoint $h$. In what follows we can work with isometries or coisometries, but for convenience of notation we restrict ourselves to the following case:

(iv) $u$ is unitary.

Let $\left\{\lambda_{n}^{2}\right\}_{n \in \mathbb{Z}}$ with $0 \leq \ldots \leq \lambda_{n} \leq \lambda_{n+1} \leq \ldots$ be all the eigenvalues of $x^{*} x$. The properties $(i i)$ and $(i i i)$ imply that there exists an orthonormal basis $\left\{e_{n}\right\}_{n \in \mathbb{Z}}$ in $H$ and a bijection $m: \mathbb{Z} \rightarrow \mathbb{Z}$ such that this basis diagonalizes both $x^{*} x$ and $x x^{*}$ and one has

$$
x^{*} x e_{n}=\lambda_{n}^{2} e_{n} \quad \text { and } \quad x x^{*} e_{n}=\lambda_{m(n)}^{2} e_{n} .
$$

Received by the editors October 7, 2004 and, in revised form, March 21, 2005.

2000 Mathematics Subject Classification. Primary 47L60; Secondary 19K14, 46L80.

Key words and phrases. Bott projections, $K$-group, deformations, invariant.

The first author was supported in part by the RFFI grant No. 05-01-00923 and HW619.2003.01, and the second author by the Crafoord Foundation, the Swedish Foundation for International Cooperation in Research and Higher Education (STINT) and the Royal Swedish Academy of Sciences. Part of this research was performed during the Non-commutative Geometry program 2003/2004, Mittag-Leffler Institute, Stockholm.

(c) 2006 American Mathematical Society Reverts to public domain 28 years from publication 
Note that if all $\lambda_{n}, n \in \mathbb{Z}$, are distinct, then the bijection $m$ is unique. Otherwise different choices of a basis above may result in different bijections $m: \mathbb{Z} \rightarrow \mathbb{Z}$, but finite multiplicity of all eigenvalues implies that all bijections satisfying (10) behave in the same way at infinity. We will also assume one more property, which does not depend on a choice of $m$ satisfying (1):

(v) $m(-\infty)=-\infty$ and $m(\infty)=\infty$, i.e. $\lim _{n \rightarrow \pm \infty} m(n)= \pm \infty$.

One can look at such an operator $x$ as at a deformed plane: if $u$ commutes with $h$ and if $\operatorname{Sp} u=\mathbb{T}, \operatorname{Sp} h=[0, \infty)$ then continuous functions of $x$ are in oneto-one correspondence with continuous functions on a plane. The $K$-group of a plane, $K_{0}\left(\mathbb{R}^{2}\right)$, is a free abelian group generated by the Bott element, i.e. by the formal difference $[p]-[q], p, q \in M_{2}\left(C_{0}\left(\mathbb{R}^{2}\right)^{+}\right)$(here the superscript + denotes the unitalization), where $p$ is a Bott projection and $q=\left(\begin{array}{ll}1 & 0 \\ 0 & 0\end{array}\right)$. To measure the deviation of non-commutative deformations of topological spaces from their 'commutative' originals, one deforms the Bott element (i.e. one changes functions involved in the commuting projections $p$ and $q$ by their non-commutative analogs - expressions in $x^{*} x$ and $x x^{*}$, which result in projections $P$ and $Q$ ) and checks its non-triviality. For the case of compact spaces like a sphere or a torus, see [6, 4], where it was shown that the invariant $\operatorname{tr}(P-Q)$ is an integer, which vanishes in the commutative case and which equals one for the Voiculescu pair (a non-commutative version of a two-torus). The same approach works for non-compact spaces as well, as pointed out in [3, 5], where it was shown that a similar invariant equals one for a 'non-commutative plane' given by a pair of self-adjoint unbounded operators $(A, B)$ that is a 'small' deformation of the pair $\left(x, i \frac{d}{d x}\right)$.

For the complex coordinate $z$ on a plane, one can use, for a Bott projection, the formula

$$
p=\left(\begin{array}{cc}
f\left(|z|^{2}\right) & g\left(|z|^{2}\right) \bar{z} \\
g\left(|z|^{2}\right) z & 1-f\left(|z|^{2}\right)
\end{array}\right),
$$

where $f:[0, \infty) \rightarrow[0,1)$ is continuous and increasing and $g$ is defined by $g(t)=$ $\sqrt{\frac{f(t)-f^{2}(t)}{t}}$ (thus $f$ cannot be arbitrary and should satisfy continuity of the function $\frac{f(t)-f^{2}(t)}{t}$ and its vanishing at 0$)$.

Passing to the non-commutative framework, one should consider, for an operator $x$ with properties $(i)-(v)$, the operator

$$
P=P_{f}(x)=\left(\begin{array}{ll}
p_{11} & p_{12} \\
p_{21} & p_{22}
\end{array}\right)=\left(\begin{array}{cc}
f\left(x^{*} x\right) & g\left(x^{*} x\right) x^{*} \\
x g\left(x^{*} x\right) & 1-f\left(x x^{*}\right)
\end{array}\right) .
$$

As $x h\left(x^{*} x\right)=h\left(x x^{*}\right) x$ for any continuous function $h(t)$ with the property $h(0)=$ $\lim _{t \rightarrow \infty} h(t)=0$, one easily checks that $\left(P_{f}(x)\right)^{2}=P_{f}(x)$, i.e. the operator $P_{f}(x)$ is a projection.

Let $C(H)$ denote the set of trace class operators on $H$ and let $\chi_{(\varepsilon, \infty)}$ denote the characteristic function of the interval $(\varepsilon, \infty)$ for some $\varepsilon>0$. Put $q_{\varepsilon}=\chi_{(\varepsilon, \infty)}\left(x^{*} x\right)$. Due to the property (ii), one can choose the function $f$ such that $p_{11}-q_{\varepsilon} \in C(H)$ and $p_{12}, p_{21} \in C(H)$, and due to the properties $(i i)$ and $(v)$, the function $f$ can be chosen in such a way that $p_{22}-\left(1-q_{\varepsilon}\right) \in C(H)$. Summing up the properties of an admissible function $f:[0, \infty) \rightarrow[0,1)$, we have:

a) $f(0)=0, \lim _{t \rightarrow \infty} f(t)=1$ and the function $g(t)=\sqrt{\frac{f(t)-f^{2}(t)}{t}}$ is continuous with $g(0)=0$; 
b) the series $\sum_{n>0}\left(f\left(\lambda_{n}^{2}\right)-1\right)$ and $\sum_{n<0} f\left(\lambda_{n}^{2}\right)$ are square summable, where $\lambda_{n}^{2}$ are the eigenvalues of $x^{*} x$.

Note that these properties imply $f\left(x^{*} x\right)-q_{\varepsilon} \in C(H)$ and $p_{12}, p_{21} \in C(H)$. Together with $(v)$, they imply $p_{22}-\left(1-q_{\varepsilon}\right) \in C(H)$.

Define a projection $Q$ by

$$
Q=\left(\begin{array}{cc}
q_{\varepsilon} & 0 \\
0 & 1-q_{\varepsilon}
\end{array}\right)
$$

After an appropriate choice of $f$ among functions satisfying a) and b) one has $P_{f}(x)-Q \in C(H \oplus H)$.

Definition 1. Put $\omega(x)=\operatorname{tr}\left(P_{f}(x)-Q\right)$.

To prove that $\omega(x)$ is well defined, we require the following well-known lemma. Its proof can be found, e.g. in [2, Lemma VII.8.5.

Lemma 2. Let $P$ and $Q$ be projections in $B(H)$ with $P-Q \in C(H)$. Then $\operatorname{tr}(P-Q)$ is an integer.

Theorem 3. The number $\omega(x)$ is a well-defined integer and does not depend on the choice of a bijection $m$ obeying (1), of $\varepsilon$ and of a function $f$ obeying a) and b).

Proof. Since $P_{f}(x)$ and $Q$ are projections with $P_{f}(x)-Q \in C(H \oplus H), \operatorname{tr}\left(P_{f}(x)-Q\right)$ is an integer by Lemma 2 .

Independence from the choice of $m$ and of $\varepsilon$ is obvious. To check that $\operatorname{tr}\left(P_{f}(x)-Q\right)$ does not depend on our choice of an admissible function $f$, take two such functions, $f$ and $f^{\prime}$. Then the function $f_{s}=s f+(1-s) f^{\prime}, s \in[0,1]$, also satisfies a) and b), hence $P_{f_{s}}(x)-Q \in C(H \oplus H)$. Continuity of $\operatorname{tr}\left(P_{f_{s}}(x)-Q\right)$ with respect to the parameter $s$ completes the proof.

Below we calculate $\omega(x)$ in some cases. We take $H=l_{2}(\mathbb{Z})$ and use the standard basis $\left\{e_{n}\right\}_{n \in \mathbb{Z}}$ for $H$. Let $L_{N} \subset H$ be a subspace generated by $e_{n},-N \leq n \leq N$. Remark that, since $P-Q \in C(H \oplus H)$, in order to calculate $\omega(x)$ it suffices to calculate $\operatorname{tr}\left(\left.(P-Q)\right|_{L_{N} \oplus L_{N}}\right)$ for big enough $N$.

Example 4. Let $x$ be normal of the form $x e_{n}=\lambda_{n} e_{n}$, where $0 \leq \ldots \leq \lambda_{n} \leq$ $\lambda_{n+1} \leq \ldots, \lim _{n \rightarrow-\infty} \lambda_{n}=0, \lim _{n \rightarrow \infty} \lambda_{n}=\infty$. Then obviously $\operatorname{tr}\left(\left.P\right|_{L_{N} \oplus L_{N}}\right)=$ $\operatorname{tr}\left(\left.Q\right|_{L_{N} \oplus L_{N}}\right)=N$, hence $\omega(x)=0$.

Example 5. Let $x$ be of the form $x e_{n}=\lambda_{n} e_{n+1}$, where $0 \leq \ldots \leq \lambda_{n} \leq \lambda_{n+1} \leq \ldots$, $\lim _{n \rightarrow-\infty} \lambda_{n}=0, \lim _{n \rightarrow \infty} \lambda_{n}=\infty$. Then $x^{*} x e_{n}=\lambda_{n}^{2} e_{n}, x x^{*} e_{n}=\lambda_{n-1}^{2} e_{n}$, and

$$
\operatorname{tr}\left(\left.P\right|_{L_{N} \oplus L_{N}}\right)=\sum_{n=-N}^{N} f\left(\lambda_{n}^{2}\right)+\sum_{n=-N}^{N}\left(1-f\left(\lambda_{n-1}^{2}\right)\right)=N-f\left(\lambda_{-N-1}^{2}\right)+f\left(\lambda_{N}^{2}\right) .
$$

On the other hand, $\operatorname{tr}\left(\left.Q\right|_{L_{N} \oplus L_{N}}\right)=N$. Hence

$$
\lim _{N \rightarrow \infty} \operatorname{tr}\left(\left.(P-Q)\right|_{L_{N} \oplus L_{N}}\right)=\lim _{N \rightarrow \infty} f\left(\lambda_{N}^{2}\right)-f\left(\lambda_{-N-1}^{2}\right)=1,
$$

therefore, $\omega(x)=1$. 
Example 6. Let $x$ be of the form $x e_{n}=\lambda_{n} e_{n+k}$, where $0 \leq \ldots \leq \lambda_{n} \leq \lambda_{n+1} \leq \ldots$, $\lim _{n \rightarrow-\infty} \lambda_{n}=0, \lim _{n \rightarrow \infty} \lambda_{n}=\infty$. Then $x^{*} x e_{n}=\lambda_{n}^{2} e_{n}, x x^{*} e_{n}=\lambda_{n-k}^{2} e_{n}$, and

$$
\begin{aligned}
\operatorname{tr}\left(\left.P\right|_{L_{N} \oplus L_{N}}\right) & =\sum_{n=-N}^{N} f\left(\lambda_{n}^{2}\right)+\sum_{n=-N}^{N}\left(1-f\left(\lambda_{n-1}^{2}\right)\right) \\
& =N-\sum_{i=1}^{k} f\left(\lambda_{-N-i}^{2}\right)+\sum_{i=1}^{k} f\left(\lambda_{N-i+1}^{2}\right) .
\end{aligned}
$$

Once more, $\operatorname{tr}\left(\left.Q\right|_{L_{N} \oplus L_{N}}\right)=N$. Hence

$$
\lim _{N \rightarrow \infty} \operatorname{tr}\left(\left.(P-Q)\right|_{L_{N} \oplus L_{N}}\right)=\lim _{N \rightarrow \infty} \sum_{i=1}^{k} f\left(\lambda_{N-i+1}^{2}\right)-\sum_{i=1}^{k} f\left(\lambda_{-N-i}^{2}\right)=k,
$$

therefore, $\omega(x)=k$.

Example 7. Important classes of examples are obtained from Example 5 when $\lambda_{n}^{2}$ belongs to orbits of dynamical systems for all $n$, or more precisely when $\lambda_{n+1}^{2}=$ $F\left(\lambda_{n}^{2}\right), n \in \mathbb{Z}$, in Example 5, where $F: \mathbb{R} \rightarrow \mathbb{R}$ is a Borel measurable mapping of the real line. Under conditions of Example 5 the operator $x$ satisfies the commutation relation $x^{*} x=F\left(x^{*} x\right)$ as

$$
x^{*} x e_{n}=\lambda_{n}^{2} e_{n}=F\left(\lambda_{n-1}^{2}\right) e_{n}=F\left(x x^{*}\right) e_{n},
$$

where the spectral mapping theorem was used in the last equality. For example, if $F(t)=q t$ for $q>1$, then $\lambda_{n}=\sqrt{F^{\circ n}\left(\lambda_{0}^{2}\right)}=\sqrt{q^{n} \lambda_{0}}, n \in \mathbb{Z}$, satisfies the conditions of Example 5 for any starting point $\lambda_{0}>0$. The operator $x$ satisfies the 'quantum plane' commutation relation $x^{*} x=q x x^{*}$. By the result in Example 5 we have $\omega(x)=1$.

Now we are going to give an interpretation of $\omega(x)$ in $K$-theory terms. Let $h^{\prime}$ be the unbounded operator on $H$ given by $h^{\prime} e_{n}=\mu_{n} e_{n}$, where $\left\{e_{n}\right\}$ is the eigenbasis of $h$ and the eigenvalues $0 \leq \ldots \leq \mu_{n} \leq \mu_{n+1} \leq \ldots$ have the same multiplicity as those of $h$, i.e. $\mu_{i}=\mu_{j}$ iff $\lambda_{i}=\lambda_{j}$ and $\mu_{n}=0$ iff $\lambda_{n}=0$. Put $y=u h^{\prime}$. Then $y$ evidently satisfies the properties $(i)-(v)$ except $(i i i)$, and the latter property can be checked as follows.

Since $x^{*} x e_{n}=h^{2} e_{n}=\lambda_{n}^{2} e_{n}$ and $x x^{*} e_{n}=u h^{2} u^{*} e_{n}=\lambda_{m(n)}^{2} e_{n}$, i.e. $h^{2} u^{*} e_{n}=$ $\lambda_{m(n)}^{2} u^{*} e_{n}$, one has $u^{*} e_{n} \in \operatorname{Span}\left\{e_{i}: \lambda_{i}=\lambda_{m(n)}\right\}$, hence $\left(h^{\prime}\right)^{2} u^{*} e_{n}=\mu_{m(n)}^{2} u^{*} e_{n}$ by definition of $h^{\prime}$. Therefore, $u\left(h^{\prime}\right)^{2} u^{*}$ is diagonal with respect to the basis $\left\{e_{n}\right\}_{n \in \mathbb{Z}}$. This implies that $y^{*} y=\left(h^{\prime}\right)^{2}$ and $y y^{*}=u\left(h^{\prime}\right)^{2} u^{*}$ commute.

Now consider the non-trivial case, when $h$ is not the identity operator. Then there is a number $i$ such that $\lambda_{i-1}<\lambda_{i}$, and without loss of generality (by renumbering) we may assume that $i=0$. Let $\widetilde{h}$ be the unbounded operator on $H$ given by $\widetilde{h} e_{n}=\mu_{n} e_{n}$, where $\left\{e_{n}\right\}$ is the eigenbasis of $h$ and the eigenvalues $0 \leq \ldots \leq \mu_{n} \leq \mu_{n+1} \leq \ldots$ are given by $\mu_{n}=\lambda_{n}$ for $n \geq 0$ and $\mu_{n}=0$ for $n<0$. Set $\mu_{n}(t)=\frac{1}{t+1} \lambda_{n}$ for $n<0$ and $\mu_{n}(t)=\lambda_{n}$ for $n \geq 0$ and define a family of unbounded operators $\left(\widetilde{h}_{t}\right)_{t \in[0, \infty)}$ by $\widetilde{h}_{t} e_{n}=\mu_{n}(t) e_{n}$. Also, set $z=u \widetilde{h}, z_{t}=u \widetilde{h}_{t}$. Although both $z_{t}$ and $z$ are unbounded, their difference is bounded and diagonal, and one has $\lim _{t \rightarrow \infty}\left\|z_{t}-z\right\|=\lim _{t \rightarrow \infty} \sup _{n<0}\left|\frac{\lambda_{n}}{t+1}\right|=\lim _{t \rightarrow \infty}\left|\frac{\lambda_{-1}}{t+1}\right|=0$. By the previous argument $z_{t}$ satisfies the property (iii) for each $t$, and passing to the limit 
as $t \rightarrow \infty$ one sees that $(i i i)$ holds for $z$ as well. Properties $(i),(i i),(i v)$ and $(v)$ obviously hold for $z$.

Lemma 8. One has $\omega(x)=\omega(y)=\omega(z)$.

Proof. Put $x(t)=(1-t) x+t y$. Then $\omega(x(t))$ is continuous. But it is integer-valued, hence constant. The argument for $z$ is the same.

Lemma 9. There exists a one-parameter family $\left(\alpha_{t}\right)_{t \in(0, \infty)}$ of homeomorphisms of $\mathbb{R}_{+}$such that

$$
\lim _{t \rightarrow \infty}\left\|u \varphi\left(\alpha_{t}(h)\right)-\varphi\left(\alpha_{t}(h)\right) u\right\|=0
$$

for any $\varphi \in C_{0}([0, \infty))$.

Proof. There exists a sequence $\left(\mu_{n}\right)_{n \in \mathbb{Z}}$ such that $0 \leq \ldots \leq \mu_{-1} \leq \mu_{0} \leq \mu_{1} \leq \mu_{2} \leq$ $\ldots$ such that $\mu_{i}=\mu_{j}$ iff $\lambda_{i}=\lambda_{j}$ and $\mu_{n}=0$ iff $\lambda_{n}=0$ and $\left|\mu_{m(n)}-\mu_{n}\right| \leq 1$ for all $n$. Take a homeomorphism $\psi: \mathbb{R}_{+} \rightarrow \mathbb{R}_{+}$such that $\psi\left(\lambda_{n}\right)=\mu_{n}$ and let $\beta_{t}(s)=\frac{s}{t+1}, t>0$. Put $\alpha_{t}=\psi^{-1} \circ \beta_{t} \circ \psi$ and $\widetilde{\varphi}=\varphi \circ \psi^{-1}$. Then

$$
\begin{aligned}
& \left\|u \varphi\left(\alpha_{t}(h)\right)-\varphi\left(\alpha_{t}(h)\right) u\right\|=\sup _{n \in \mathbb{Z}}\left|\varphi\left(\alpha_{t}\left(\lambda_{m(n)}\right)\right)-\varphi\left(\alpha_{t}\left(\lambda_{n}\right)\right)\right| \\
& =\sup _{n \in \mathbb{Z}}\left|\varphi\left(\beta_{t}\left(\mu_{m(n)}\right)\right)-\varphi\left(\beta_{t}\left(\mu_{n}\right)\right)\right|=\sup _{n \in \mathbb{Z}}\left|\widetilde{\varphi}\left(\frac{\mu_{m(n)}}{t+1}\right)-\widetilde{\varphi}\left(\frac{\mu_{n}}{t+1}\right)\right|,
\end{aligned}
$$

and (3) holds because $\sup _{n \in \mathbb{Z}}\left|\frac{\mu_{m(n)}}{t+1}-\frac{\mu_{n}}{t+1}\right| \leq \frac{1}{t+1}$ vanishes as $t \rightarrow \infty$ and because $\widetilde{\varphi} \in C_{0}([0, \infty))$, hence is uniformly continuous.

Theorem 10. $\omega(x)=\operatorname{index}\left(u q_{\varepsilon}\right)$.

Proof. Without loss of generality we can assume that $f(r)=0$ for $r \in(0, \varepsilon)$. Then $\left(\begin{array}{cc}q_{\varepsilon} & 0 \\ 0 & q_{\varepsilon}\end{array}\right)$ commutes with $P_{f}(x)$ and with $Q$ and $\left(\begin{array}{cc}1-q_{\varepsilon} & 0 \\ 0 & 1-q_{\varepsilon}\end{array}\right)\left(P_{f}(x)-Q\right)=0$, hence

$$
\omega(x)=\operatorname{tr}\left(\left(\begin{array}{cc}
q_{\varepsilon} & 0 \\
0 & q_{\varepsilon}
\end{array}\right) P_{f}(x)-\left(\begin{array}{cc}
q_{\varepsilon} & 0 \\
0 & 0
\end{array}\right)\right) .
$$

Let $y=u k$, where $k=\sum_{n \in \mathbb{Z}} \mu_{n} e_{n}$, where $\mu_{n}=0$ for $n<0$ and $\mu_{n}=\lambda_{n}$ for $n \geq 0$. By Lemma 8, $\omega(y)=\omega(x)$. Let $H_{0}=q_{0}(H)$ and let $u_{0}=u q_{0}, k_{0}=q_{0} k$, $y_{0}=u_{0} k_{0}$. All these operators and all further formulas are in the Hilbert space $H_{0}$. The formula for $\omega\left(y_{0}\right)$ in $H_{0}$ is written as $\omega\left(y_{0}\right)=\operatorname{tr}\left(P_{f}\left(y_{0}\right)-\left(\begin{array}{ll}0 & 0 \\ 0 & 1\end{array}\right)\right)$, and it follows from (4) that $\omega\left(y_{0}\right)=\omega(x)$.

Let $C_{0}\left(\mathbb{R}^{2}\right)$ be the $C^{*}$-algebra of continuous functions on $\mathbb{R}^{2}$ vanishing at infinity. This algebra is generated by the functions $e^{2 \pi i \theta}$ and $\varphi(r), \varphi \in C_{0}([0, \infty))$, where $\theta$ and $r$ are the polar coordinates on the plane. Note also that the operator $u \varphi(h)$ is compact for any $\varphi \in C_{0}([0, \infty))$. In what follows the main tool will be $E$-theory of Connes and Higson [1] based on the notion of asymptotic homomorphisms. Recall that an asymptotic homomorphism from a $C^{*}$-algebra $A$ to a $C^{*}$-algebra $B$ is a family of maps $\tau=\left(\tau_{t}\right)_{t \in[0, \infty)}: A \rightarrow B$ such that $t \rightarrow \tau_{t}(a)$ is continuous for each $a \in A$, and $\tau_{t}\left(a_{1} a_{2}\right)-\tau_{t}\left(a_{1}\right) \tau_{t}\left(a_{2}\right), \tau_{t}\left(\lambda a_{1}+a_{2}\right)-\lambda \tau_{t}\left(a_{1}\right)-\tau_{t}\left(a_{2}\right)$ and $\tau_{t}\left(a^{*}\right)-\tau_{t}(a)^{*}$ vanish as $t \rightarrow \infty$ for all $a, a_{1}, a_{2} \in A$ and all $\lambda \in \mathbb{C}$.

Corollary 11. The operator $y_{0}$ defines, by $e^{2 \pi i \theta} \mapsto u_{0}, \varphi(r) \mapsto \varphi\left(\alpha_{t}\left(k_{0}\right)\right)$, the asymptotic homomorphism $\tau^{y_{0}}=\left(\tau_{t}^{y_{0}}\right)_{t \in(0, \infty)}$ from $C_{0}\left(\mathbb{R}^{2}\right)$ to the $C^{*}$-algebra $K\left(H_{0}\right)$ of compact operators. 
Thus $x$, and then $y_{0}$, defines a class $\left[\tau^{y_{0}}\right]$ in the $E$-theory group $E\left(C_{0}\left(\mathbb{R}^{2}\right), K(H)\right)$, which is the same as the $K$-homology group of $C_{0}\left(\mathbb{R}^{2}\right)$. Let $e$ be a class in the $K$ theory group of $\mathbb{R}^{2}$. Then the pairing between $K$-theory and $K$-homology provides us with an integer $\left\langle e,\left[\tau^{y_{0}}\right]\right\rangle$.

Recall that the Bott class $\beta$ in $K_{0}\left(\mathbb{R}^{2}\right)$ is given by the formal difference of projections $p$ (2) and $\left(\begin{array}{ll}0 & 0 \\ 0 & 1\end{array}\right)$.

Lemma 12. One has $\omega\left(y_{0}\right)=\left\langle\beta,\left[\tau^{y_{0}}\right]\right\rangle$, where $\beta \in K_{0}\left(\mathbb{R}^{2}\right)$ is the Bott class.

Proof. Note that $p$ is a two-by-two matrix over the algebra $C_{0}\left(\mathbb{R}^{2}\right)$ with adjoined unit and we can extend $\tau_{t}^{y_{0}}$ onto this algebra by defining $\tau_{t}^{y_{0}}(1)=1$. Then it evidently follows from Corollary 11 that $\lim _{t \rightarrow \infty}\left\|\tau_{t}^{y_{0}}(p)-P_{f}\left(y_{0}\right)\right\|=0$. Without loss of generality we can assume that the function $f$ satisfies $f(r)=1$ for $r \in(N, \infty)$ for sufficiently large $N$. Then the operator $\tau_{t}^{y_{0}}(p)-\left(\begin{array}{ll}0 & 0 \\ 0 & 1\end{array}\right)$ is of finite rank and one has $\left\langle\beta,\left[\tau^{y_{0}}\right]\right\rangle=\lim _{t \rightarrow \infty} \operatorname{tr}\left(\tau_{t}^{y_{0}}(p)-\left(\begin{array}{ll}0 & 0 \\ 0 & 1\end{array}\right)\right)=\omega\left(y_{0}\right)$ (cf. [4]).

To finish the proof of the theorem, note that $u_{0}$ is Fredholm, hence it defines a homomorphism $\rho: C(\mathbb{T}) \rightarrow B\left(H_{0}\right) / K\left(H_{0}\right)$ and (which is the same) an extension of $C(\mathbb{T})$ by the $C^{*}$-algebra of compact operators on $H_{0}$. The class of this extension in the $K$-homology group $K_{1}(\mathbb{T})$ is given by index $\left(u_{0}\right)$. On the other hand, since the Connes-Higson construction [1] applied to the map $\rho$ gives the asymptotic homomorphism $\tau^{y_{0}}$ (this is because $(1-f)\left(\alpha_{t}\left(k_{0}\right)\right)$ is an approximate unit in $K\left(H_{0}\right)$, asymptotically commuting with $\left.u_{0}\right)$, we have index $\left(y_{0}\right)=\left\langle\beta,\left[\tau^{y_{0}}\right]\right\rangle$. Finally note that the Fredholm operators $u q_{\varepsilon}$ and $u_{0}$ differ by a finite rank operator, hence they have the same index.

\section{REFERENCES}

[1] A. Connes, N. Higson. Déformations, morphismes asymptotiques et $K$-théorie bivariante. $C$. R. Acad. Sci. Paris Sér. I Math. 311 (1990), 101-106. MR1065438(91m:46114)

[2] K. Davidson. $C^{*}$-Algebras by Example. Fields Institute Monographs, AMS, 1996. MR:1402012 (97i:46095)

[3] G. A. Elliott, T. Natsume, R. Nest. The Heisenberg group and K-theory. K-Theory 7 (1993), 409-428. MR.1255059 (94m:46109)

[4] R. Exel, T. A. Loring. Invariants of almost commuting unitaries. J. Funct. Anal. 95 (1991), 364-376. MR 1092131 (92a:46083)

[5] V. M. Manuilov. An invariant for pairs of almost commuting unbounded operators. Funktsional. Anal. i Prilozhen. 32 (1998), No. 4, 88-91 (in Russian). English translation: Functional Anal. Appl. 32 (1998), 288-291. MR1678864 (99m:47030)

[6] D. Voiculescu. Asymptotically commuting finite rank unitary operators without commuting approximants. Acta Sci. Math. (Szeged) 45 (1983), 429-431. MR0708811 (85d:47035)

Department of Mechanics and Mathematics, Moscow State University, Leninskie Gory, Moscow, 119992, Russia

E-mail address: manuilov@mech.math.msu.su

Department of Mathematics, Centre for Mathematical Sciences, Lund Institute of Technology, Lund University, P.O. Box 118, SE-221 00 Lund, Sweden

E-mail address: sergei.silvestrov@math.lth.se 